\title{
Necessary and Sufficient Conditions for Perfect Channel Shortening and Implications for Interference Mitigation
}

\author{
John M. Walsh, Richard K. Martin, Andrew G. Klein, Nicholas S. Xenias \\ Jason A. Pagnotta, and C. Richard Johnson, Jr. ${ }^{1}$ \\ School of Electrical and Computer Engineering \\ Cornell University \\ Ithaca, NY 14853-3801
}

\begin{abstract}
We explore the shortening of multiple channels arising from either over-sampling or from using multiple antennas between a single transmitter and receiver. Using terminology we develop, we give new necessary and sufficient conditions for perfect channel shortening (PCS). In an example, the perfect channel shortener's robustness against wide-band noise, narrow-band interferers, and received signal SNR mismeasurement is compared to that of an imperfect channel shortener. The work concludes with a list of possible extensions of the results and future work.
\end{abstract}

\section{INTRODUCTION}

In this paper we discuss multi-channel shortening. Specifically, we are interested in a multi-channel model which can arise from either using multiple antennas or by over-sampling between one transmitter and one receiver. We give conditions under which perfect channel shortening is possible. We focus on the maximum shortening SNR (MSSNR) solution of [1], which is equivalent to the minimum mean squared error (MMSE) solution of [2] when the source sequence is white and in the absence of noise. Neither of these papers explicitly considers the oversampled case, although [1] formulates the MSSNR problem for simultaneously shortening multiple channels in a MISO setting. No conditions for a perfect solution were given.

Fractionally-spaced channel shortening was considered in [4], [5]. Therein, the authors first assumed that the MMSE target impulse response (TIR) was set to a known vector. Then sufficient conditions were derived for perfectly achieving this known impulse response. These conditions are essentially the same as the conditions for perfect equalization. If the channels $\left\{h^{(p)}, 1 \leq p \leq P\right\}$ have at most $\nu$ common roots and these roots are included in the TIR, then there exists a non-trivial exact solution to the channel shortening problem (with a minimum length constraint on the TEQs). A proof and further results will be given in [6] and [7].

Miyajima and Ding have also considered fractionally-spaced channel shortening in [8], [9]. They state that if a given channel shortener satisfies a particular set of conditions, then it perfectly shortens the channel. However, they do not give sufficient conditions for the existence of a perfect channel shortener in terms of requirements on the length or zeros of the channel and shortener.

\footnotetext{
${ }^{1}$ This work was supported in part by NSF Grant CCR0310023, Applied Signal Technology, Texas Instruments, and the Olin Fellowship from Cornell University. Email addresses for Walsh, Klein, Xenias, Pagnotta, Martin, and Johnson are \{jmw56,agk5,ns×2,jap43\}@cornell.edu and \{frodo,johnson\}@ece.cornell.edu respectively.
}

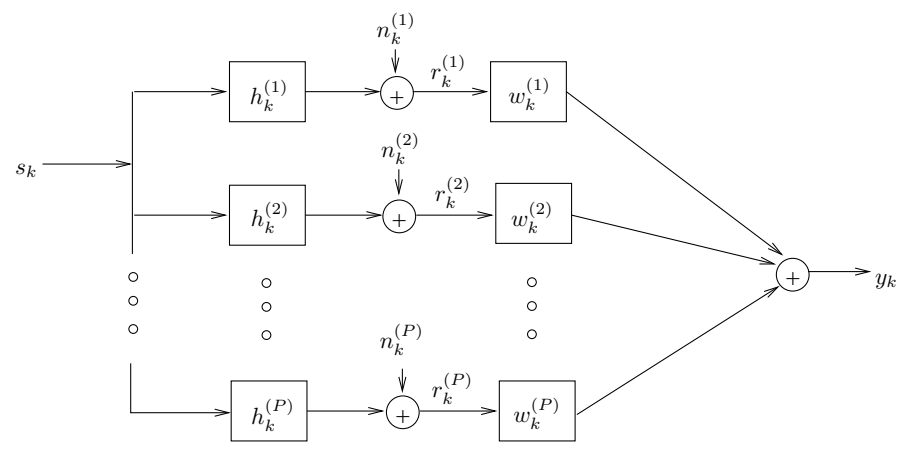

Figure 1: The system model.

Schemes for MIMO channel shortening have been proposed in [1], [10] - [15]. However, these papers primarily focus on proposing designs and quantifying their performance.

In this paper, we build a set of terminology for channel shortening, recast and prove sufficient conditions similar to those in $[3,4,6,7]$, and develop and prove novel necessary conditions for various types of perfect channel shortening. In Section II we introduce the model for the system and the notation we will use. Section III develops the definitions of various types of perfect channel shortening and gives examples of channels that motivate the definitions we have chosen. Section IV then takes the new terminology and uses it to provide necessary and sufficient conditions for the different types of perfect channel shortening. In many cases, the set of perfect channel shorteners will be a large space of possible choices. Section V helps choose which perfect channel shortener to use by investigating each perfect channel shortener's robustness to interference. We also consider the possibility of relaxing the perfect channel shortening requirement in order to obtain a higher SINR (similar to [1]). Section VI concludes the work by giving an example of two different channel shortening designs, both considering the effect of a narrowband interferer and noise. We see that both the relaxed and perfect designs have very similar performances, and we compare their ability to notch out the narrowband interferer as it varies in frequency.

\section{System Model And Notation}

We wish to study the system shown in Fig. 1. This model can arise in practice either by sampling a single bandlimited continuous time channel at $P$ times the baud rate, or by using $P$ receive antennas. $s_{k}$ is a series of transmitted data symbols. The $P$ received signals, $r_{k}^{(i)}, i \in\{1, \ldots, P\}$, are created by passing the original source sequence through $P$ different noisy linear time invariant channels. The $i$ th channel has an impulse response $h_{k}^{(i)}$ of length $L_{h}+1$ (that is, 
$\left.h_{k}^{(i)}=0 \forall k \in\{-\infty, \ldots,-1\} \cup\left\{L_{h}+1, \ldots, \infty\right\}\right)$ and noise sequence $n_{k}^{(i)}$. For any number of reasons, we may wish to shorten the overall effective impulse response between $s_{k}$ and $y_{k}$ to have non-zero taps only within a window of length $\nu+1[1],[11]$. To do this, we use a bank of linear filters called channel shorteners, the $i$ th channel shortener having an impulse response $w_{k}^{(i)}$ and length $L_{w}+1$. Denote the $p$ th sub-channel's z-transform as

$$
h^{(p)}(z)=\sum_{i=0}^{L_{h}} h_{i}^{(p)} z^{-i}
$$

and the $p$ th sub-channel's channel shortener's z-transform as $w^{(p)}(z)$ in the same manner.

$$
w^{(p)}(z)=\sum_{i=0}^{L_{w}} w_{i}^{(p)} z^{-i}
$$

We assume that the channel length $L_{h}$ has been chosen such that at least one of the sub-channels does not have zero as its first or last element. This avoids common sub-channels roots at zero or infinity. Define the $p$ th channel's convolution matrix of size $\left(L_{h}+L_{w}+1\right) \times\left(L_{w}+1\right)$

$$
\mathbf{H}_{p}=\left(\begin{array}{ccccc}
h_{0}^{(p)} & \cdots & h_{L_{h}}^{(p)} & & \\
& \ddots & & \ddots & \\
& & h_{0}^{(p)} & \cdots & h_{L_{h}}^{(p)}
\end{array}\right)^{T}
$$

and define the $p$ th channel's shortener to be $\mathbf{w}_{p}=$ $\left(w_{0}^{(p)}, w_{1}^{(p)}, \ldots, w_{L_{w}}^{(p)}\right)^{T}$. By concatenating the channel convolution matrices to form $\mathbf{H}=\left(\mathbf{H}_{1}\left|\mathbf{H}_{2}\right| \cdots \mid \mathbf{H}_{P}\right)$, and concatenating the channel shorteners to form $\mathbf{w}=\left(\mathbf{w}_{1}^{T}\left|\mathbf{w}_{2}^{T}\right| \ldots \mid \mathbf{w}_{P}^{T}\right)^{T}$, we can write the vector overall combined impulse response between $s_{k}$ and $y_{k}$ as

$$
\mathbf{c}=\mathbf{H w}
$$

Denote $\mathbf{H}_{\text {wall }}(\Delta, \nu)$ to be the matrix remaining after removing the $\Delta$ th to the $\Delta+\nu$ th rows from $\mathbf{H}$, and denote $\mathbf{H}_{\text {win }}(\Delta, \nu)$ to be the $\Delta$ th to the $\Delta+\nu$ th rows of $\mathbf{H}$. These matrices will be useful in our definition of channel shortening terminology.

\section{TERMinOLOGY}

One can speak of shortening channels to different lengths, the ability to shortening them at many or a few delays, and the ability to obtain many or only a few impulse responses within the window. In order to discuss these different topics in a precise manner, we now introduce some mathematical terminology to name each of these different types of channel shortening.

Definition III.1 ( $\nu$-PCS) w is a perfect channel shortener of window length $\nu+1(\nu-P C S)$ and delay $\Delta \in\left\{0,1, \ldots, L_{h}+L_{w}-\right.$ $\nu\}$ for a channel $\mathbf{h}$ if $\mathbf{H w} \neq \mathbf{0}$ and $\mathbf{H}_{\text {wall }}(\Delta, \nu) \mathbf{w}=\mathbf{0}$.

Definition III.2 (Strong $\nu$-PCS Property) A channel $\mathbf{h}$ has the strong $\nu$-PCS property if for every delay $\Delta \in$ $\left\{0,1, \ldots, L_{h}+L_{w}-\nu\right\}$ there exists a $\mathbf{w}$ which is a $\nu$-PCS.

Definition III.3 (Weak $\nu$-PCS Property) A channel $\mathbf{h}$ has the weak $\nu$-PCS property if there exists at least one delay $\Delta \in\left\{0,1, \ldots, L_{h}+L_{w}-\nu\right\}$ for which there is a $\nu$-PCS.
Definition III.4 (M-flexible $\nu$-PCS Property) A channel $\mathbf{h}$ has the $M$-flexible $\nu$-PCS Property if there exist $M$ linearly independent vectors $\mathbf{c}_{1}, \mathbf{c}_{2}, \cdots, \mathbf{c}_{M}$ each in $\mathbb{R}^{L_{h}+L_{w}+1}$ and $M$ linearly independent vectors $\mathbf{w}_{1}, \mathbf{w}_{2}, \cdots, \mathbf{w}_{M}$ each in $\mathbb{R}^{L_{w+1}}$, such that every $\nu$-PCS, $\mathbf{w}$, has a shortened channel, $\mathbf{H w}$, that is a linear combination of $\mathbf{c}_{1}, \mathbf{c}_{2}, \cdots, \mathbf{c}_{M}$, and every linear combination of $\mathbf{w}_{i}$ 's is a $\nu$-PCS.

Furthermore, we say a channel has the inflexible $\nu$-PCS property or the flexible $\nu$-PCS property if it is M-flexible with $\mathrm{M}$ equal to 1 or $\nu+1$ respectively.

In order to highlight the importance of the terminology, we give two examples of low order channels that differentiate between the properties. In both examples $P=2$.

Example 1 The two sub-channels $h^{(1)}(z)=z^{-2}+2 z^{-1}+3$ and $h^{(2)}(z)=4 z^{-2}+5 z^{-1}+6$ have the strong flexible $\nu$-PCS for both $\nu=0$ and $\nu=1$ for $L_{w}=2$. As we shall see later in Section IV, this is because $h^{(1)}(z)$ and $h^{(2)}(z)$ share no common roots.

Example 2 The two sub-channels $h^{(1)}(z)=z^{-6}(z-1)(z-$ $2)(z-3)(z-4)(z-5)(z-6)$ and $h^{(2)}(z)=z^{-2}(z-1)(z-7)$ share a common root at $z=1$. They do not have any 0 -PCS properties for any $L_{w}$. They have the strong inflexible 1-PCS property for $L_{w}=4$ for reasons we will see in Section IV. They only have the weak inflexible 2-PCS property for $L_{w}=2$ because they are shortenable for $\Delta=0$ but are not shortenable for $\Delta=5$.

\section{Conditions}

Now that we have defined and clarified our terminology, we are ready to provide novel necessary conditions and sufficient conditions for strong M-flexible $\nu$-PCS. We begin with some novel necessary conditions.

Theorem IV.1 (Necessary Conditions) Let the subchannels $h^{(p)}(z)$ share $\mu$ roots ${ }^{1}$. Then necessary conditions for $\mathbf{h}$ to have the Strong and $M$-flexible $\nu$-PCS property are

$$
\begin{aligned}
& \text { - } M \leq \nu-\mu+1 \\
& \text { - } \nu \geq \mu \\
& \text { - } P L_{w}+P \geq L_{h}+L_{w}-2 \nu+2 M+\mu-1
\end{aligned}
$$

Proof: First of all, note that common sub-channel roots must be included in the shortened channel. This is because

$$
h^{(p)}\left(z_{0}\right)=0 \quad \forall p \Rightarrow \sum_{p=1}^{P} h^{(p)}\left(z_{0}\right) w^{(p)}\left(z_{0}\right)=0 \quad \forall w^{(p)}(z)
$$

This implies that $M \leq \nu-\mu+1$, because in a window of length $\nu+1$ we can have at most $\nu+1$ degrees of freedom. Factor out the $\mu$ common roots from each of the $P$ sub-channels, $h^{(p)}(z)$ to form $\hat{h}^{(p)}(z)$. Thus, we now have a problem where the shortened channel can be written as

$$
c(z)=\hat{c}(z) \mu(z)
$$

where $\mu(z)$ includes only the common sub-channel roots, and we must design

$$
\hat{c}(z)=\sum_{p=1}^{P} \hat{h}^{(p)}(z) w^{(p)}(z)
$$

\footnotetext{
${ }^{1}$ These common sub-channel roots are neither at zero or infinity, otherwise we would have zero coefficients at the beginning or end of every channel, which would violate the minimality of the channel representation we choose.
} 
The second condition comes from this equation. Since we need to shorten the channel, we can not have $\nu<\mu$.

Note that we must choose $\hat{c}(z)$ to be length $M$, and due to the need to get all of the different delays, this implies we need at least $L_{h}+L_{w}-2 \nu+2 M+\mu-1$ different linearly independent c's. To see this, note that there are $L_{h}+L_{w}+1-\nu$ different values of $\Delta$, each of which corresponding to a different window within which the channel must be shortened. The minimal length we could possibly shorten the channel to and still have $M$-flexibility is $M+\mu$. Even if we could perfectly shorten the channel to the minimal length possible, we would still need $L_{h}+L_{w}+$ $1-\nu-(\nu+1-(M+\mu))=L_{h}+L_{w}-2 \nu+M+\mu$ different locations of this smaller $M+\mu$ length window to satisfy $\nu$-PCS at $L_{h}+L_{w}+1-\nu$ different values of $\Delta$. Since we needed $M$ different linearly independent c's for the first window, and we already included one of them as the first $M+\mu$ window positioning, we need a minimum of $L_{h}+L_{w}-2 \nu+M+\mu+(M-1)=$ $L_{h}+L_{w}-2 \nu+2 M+\mu-1$ different linearly independent c's. To do this, the rank of the matrix $\hat{\mathbf{H}}$, formed as $\mathbf{H}$ was formed by replacing $h_{n}^{(p)}$ with $\hat{h}_{n}^{(p)}$, must satisfy

$$
\operatorname{rank}(\hat{\mathbf{H}}) \geq L_{h}+L_{w}-2 \nu+2 M+\mu-1
$$

The rank of $\hat{\mathbf{H}}$ is less than or equal to the minimum of its two dimensions

$$
\operatorname{rank}(\hat{\mathbf{H}}) \leq \min \left(L_{h}-\mu+L_{w}+1, P L_{w}+P\right)
$$

Combining (2) and (3) we have

$\min \left(L_{h}-\mu+L_{w}+1, P L_{w}+P\right) \geq L_{h}+L_{w}-2 \nu+2 M+\mu-1$

which implies

$$
P L_{w}+P \geq L_{h}+L_{w}-2 \nu+2 M+\mu-1
$$

which is the third necessary condition.

Theorem IV.2 (Sufficient Conditions) As before, let the sub-channels $h^{(p)}(z)$ share $\mu$ (non-zero) roots. Sufficient conditions for $\mathbf{h}$ to have the Strong and $M$-flexible $\nu$-PCS property are

$$
\begin{aligned}
& \text { - } M=\nu-\mu+1 \\
& \text { - } \nu \geq \mu \\
& \text { - } P L_{w}+P \geq L_{h}+L_{w}+1-\mu
\end{aligned}
$$

Proof: Assume that the three conditions hold. Factor out the $\mu$ common sub-channel roots from each of the $h^{(p)}(z)$ to form $\hat{h}^{(p)}(z)$ for all $p \in\{1, \ldots, P\}$. The common sub-channel roots will be included in the shortened channel. Now our shortened channel is of the form (1) where none of the sub-channels $\hat{h}^{(p)}(z)$ have any common zeros and the order of the sub-channels $\hat{L}_{h}=L_{h}-\mu$ satisfies $P L_{w}+P \geq \hat{L}_{h}+L_{w}+1$. With (1), these two conditions satisfy the requirements for Strong Perfect Equalization [16], which, due to linearity, implies that we can form any $\hat{c}(z)$ we choose up to length $L_{h}-\mu+L_{w}+1$. Thus, our combined response is any channel of the form

$$
c(z)=\hat{c}(z) \mu(z)
$$

Where $\mu(z)$ is the product of common sub-channel zeros of the original channel and $\hat{c}(z)$ is an arbitrary filter. In order to perfectly shorten the channel to length $\nu+1$ we choose $\hat{c}(z)$ of length $\hat{L}_{h}=\nu-\mu+1$. This implies that $M=\nu-\mu+1$.

Here are some remarks about these two sets of conditions
Remark 1 The necessary conditions and sufficient conditions we have provided collapse into the conditions for strong perfect equalization when $\nu=0$ and $\mu=0$.

Remark 2 When we choose $M$ to be its maximum possible value, the necessary conditions are the same as the sufficient conditions. Thus, given that $M=\nu-\mu+1$, the sufficient conditions given are actually both necessary and sufficient.

Remark 3 (The Space of $\nu$-PCSs) Note that for a channel that has the M-flexible $\nu$-PCS property, the space, $\mathcal{N}$, of all $\nu$-PCSs is not a vector space. We can express $i t^{2}$ as $\mathcal{N}=$ $\operatorname{null}\left(\mathbf{H}_{\text {wall }}\right) \cap \operatorname{null}(\mathbf{H})^{c}$, and our definition of the $M$-flexible $\nu$ PCS property allows us to write it in a new way:

$$
\mathcal{N}=a_{1} \mathbf{w}_{1}+\cdots+a_{M} \mathbf{w}_{M}+\beta_{1} \mathbf{v}_{1}+\cdots+\beta_{K} \mathbf{v}_{K}
$$

Where:

- $\mathbf{w}_{1}, \ldots, \mathbf{w}_{M}$ are the linearly independent vectors referred to in the definition of the M-flexible $\nu$-PCS property.

- The $a_{i} s$ are real numbers $\left(a_{i} \in \mathbb{R} \forall i \in\{1, \ldots, M\}\right)$, and at least one of the $a_{i} s$ must be non-zero $\left(\exists j \ni a_{j} \neq 0\right)$.

- $\left\{\mathbf{v}_{1}, \ldots, \mathbf{v}_{K}\right\}$ form a basis for $\operatorname{null}(\mathbf{H})$.

- The $\beta_{i}$ s are real numbers: $\beta_{i} \in \mathbb{R} \forall i \in\{1, \ldots, K\}$.

Note that the new sufficient conditions are different from those in [4] and [6] because the restrictions we give on the channel shortener's length are looser ( $L_{w}$ may be smaller).

\section{Robustness to Interference}

In the previous sections we have considered the interference free model in which $n_{k}^{i}=0 \forall k, i$. The M-flexible $\nu$-PCS conditions we gave allow us to choose almost any ${ }^{3}$ point within a $M+K$ dimensional space (i.e. any point in $\mathcal{N}$ from (4)) to be our channel shortener and still have perfect channel shortening. But which of these possible shortened channels should we choose? One possible answer to this problem is to allow the channel model to include noise and other interferences, and then choose the perfect channel shortener that is most robust to the interference. A natural robustness criterion is the average signal to interference and noise ratio

$$
\mathrm{SINR}=\frac{\mathrm{E}\left[|\tilde{s}|^{2}\right]}{\mathrm{E}\left[|\tilde{\eta}|^{2}\right]+\mathrm{E}\left[|\tilde{\psi}|^{2}\right]}
$$

where $\tilde{s}, \tilde{\eta}$, and $\tilde{\psi}$ are the independent and uncorrelated signal, noise, and other interference components of the output of the channel shortener, $y$, respectively. To be specific, consider a model for the interference in the $i$ th channel

$$
n_{k}^{(i)}=\eta_{k}^{(i)}+\psi_{k}^{(i)}
$$

Then, the wideband noise component of $y$ is the response of the channel shortener to the wideband noise

$$
\tilde{\eta}_{k}=\sum_{i=1}^{P} \sum_{l=0}^{L_{w}} \eta_{k-l}^{(i)} w_{l}^{(i)}
$$

and the other interference component of $y$ is the response of the channel shortener to the other interference

$$
\tilde{\psi}_{k}=\sum_{i=1}^{P} \sum_{l=0}^{L_{w}} \psi_{k-l}^{(i)} w_{l}^{(i)}
$$

\footnotetext{
${ }^{2} A^{c}$, where $A$ is a set, denotes the compliment of $A$.

${ }^{3} a_{i}=0 \forall i \in\{1, \ldots, M\}$ is not allowed.
} 
Thus,

$$
\mathrm{E}\left[\left|\tilde{\eta}_{k}\right|^{2}\right]=\mathrm{E}\left[\sum_{i=1}^{P} \sum_{l=0}^{L_{w}} \bar{\eta}_{k-l}^{(i)} \bar{w}_{l}^{(i)} \sum_{j=1}^{P} \sum_{\delta=0}^{L_{w}} \eta_{k-\delta}^{(j)} w_{\delta}^{(j)}\right]
$$

Where $\bar{\eta}_{k-l}^{(i)}$ and $\bar{w}_{l}^{(i)}$ denote the complex conjugate of $\eta_{k-l}^{(i)}$ and $w_{l}^{(i)}$ respectively. Remembering the way that we built $\mathbf{w}$, we can note that the $(i-1)\left(L_{h}+1\right)+l$ th entry in it is $w_{l}^{(i)}$. So $(5)$ can be rewritten as a quadratic form in $\mathbf{w}$.

$$
\mathrm{E}\left[\left|\tilde{\eta}_{k}\right|^{2}\right]=\mathbf{w}^{H} \mathbf{R}_{\eta} \mathbf{w}
$$

where the $(i, j)$ th element of $\mathbf{R}_{\eta}$ is

$$
\mathbf{R}_{\eta}[i, j]=\bar{\eta}_{k-i+\left\lfloor i /\left(L_{w}+1\right)\right\rfloor}^{\left(\left\lfloor i /\left(L_{w}+1\right)\right\rfloor\right)} \eta_{k-j+\left\lfloor j /\left(L_{w}+1\right)\right\rfloor}^{\left(\left\lfloor j /\left(L_{w}+1\right)\right\rfloor\right)}
$$

A parallel development for $\tilde{\psi}$ gives

$$
\mathrm{E}\left[\left|\tilde{\psi}_{k}\right|^{2}\right]=\mathbf{w}^{H} \mathbf{R}_{\psi} \mathbf{w}
$$

where the $(i, j)$ th element of $\mathbf{R}_{\psi}$ is

$$
\mathbf{R}_{\psi}[i, j]=\bar{\psi}_{k-i+\left\lfloor i /\left(L_{w}+1\right)\right\rfloor}^{\left(\left\lfloor i /\left(L_{w}+1\right)\right\rfloor\right)} \psi_{k-j+\left\lfloor j /\left(L_{w}+1\right)\right\rfloor}^{\left(\left\lfloor j /\left(L_{w}+1\right)\right\rfloor\right)}
$$

We wish to choose the perfect channel shortener which maximizes the SINR.

$$
\mathbf{w}_{\text {opt }}=\arg \max _{\mathbf{w} \in \mathcal{N}} \frac{\mathbf{w}^{T} \mathbf{H}_{w i n}^{T} \mathbf{H}_{w i n} \mathbf{w}^{T}}{\mathbf{w}_{i} \mathbf{w}+\mathbf{w}^{T} \mathbf{R}_{n} \mathbf{w}}
$$

Forming the matrix $\mathbf{A}=\left(\mathbf{w}_{1}, \mathbf{w}_{2}, \ldots, \mathbf{w}_{M}, \mathbf{v}_{1}, \ldots, \mathbf{v}_{K}\right)$, we can write our set of perfect channel shorteners as $\mathbf{A b}$, for some vector $\mathbf{b} \in \mathbb{R}^{M+K}$, where the only restriction is that at least one of the first $M$ elements of $\mathbf{b}$ must be non-zero. This allows us to rewrite the optimization problem, (6), as

$$
\mathbf{w}_{\text {opt }}=\mathbf{A} \mathbf{b}_{\text {opt }}
$$

where

$$
b_{o p t}=\arg \min _{\mathbf{b}} \frac{\mathbf{b}^{T} \mathbf{A}^{T} \mathbf{H}_{w i n}^{T} \mathbf{H}_{w i n} \mathbf{A} \mathbf{b}}{\mathbf{b}^{T}\left(\mathbf{A}^{T} \mathbf{R}_{\eta} \mathbf{A}+\mathbf{A}^{T} \mathbf{R}_{\psi} \mathbf{A}\right) \mathbf{b}}
$$

This is the generalized Raleigh quotient problem, whose solution involves the generalized eigenvector problem [17]

$$
\mathbf{A}^{T} \mathbf{H}_{w i n}^{T} \mathbf{H}_{w i n} \mathbf{A} \mathbf{b}_{i}=\lambda_{i}\left(\mathbf{A}^{T} \mathbf{R}_{i} \mathbf{A}+\mathbf{A}^{T} \mathbf{R}_{n} \mathbf{A}\right) \mathbf{b}_{i}
$$

where we seek the eigenvector $\mathbf{b}_{i}$ associated with the maximum eigenvalue, $\lambda_{\max }$. Note that we have taken the cavalier attitude that we will ignore the restriction on $\mathbf{b}$ while performing the maximization. We will merely check that the maximum, $\mathbf{b}_{\text {opt }}$, of the generalized eigenvalue problem is nonzero in one of its first $M$ components. If it is not, we would have to resort to constrained optimization methods.

In some situations we may wish to relax the requirement that the channel be perfectly shortened in favor of a better SINR. In this case, in the spirit of the MSSNR design [1], we include in the SINR the out-of-window energy from the shortened channel, and optimize the ratio

$$
\mathrm{SINR}=\frac{\mathbf{w}^{T} \mathbf{H}_{\text {win }}^{T} \mathbf{H}_{\text {win }} \mathbf{w}}{\mathbf{w}^{T}\left(\mathbf{H}_{\text {wall }}^{T} \mathbf{H}_{\text {wall }}+\mathbf{R}_{i}+\mathbf{R}_{n}\right) \mathbf{w}}
$$

which, as before, leads to the generalized eigenvector problem

$$
\mathbf{H}_{w i n}^{T} \mathbf{H}_{\text {win }} \mathbf{v}_{i}=\lambda_{i}\left(\mathbf{H}_{\text {wall }}^{T} \mathbf{H}_{\text {wall }}+\mathbf{R}_{i}+\mathbf{R}_{n}\right) \mathbf{v}_{i}
$$

and we choose $\mathbf{w}=\mathbf{v}_{i}$, where $\lambda_{i}$ is the maximum eigenvalue. Note that $\mathbf{w}$ need not be in $\mathcal{N}$ here.

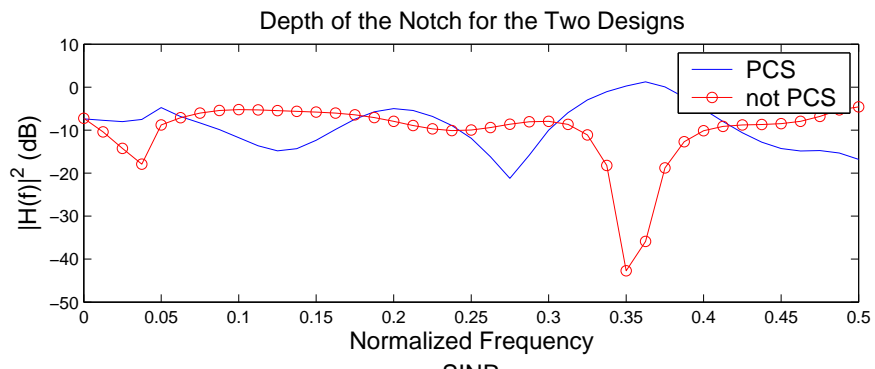

SINR

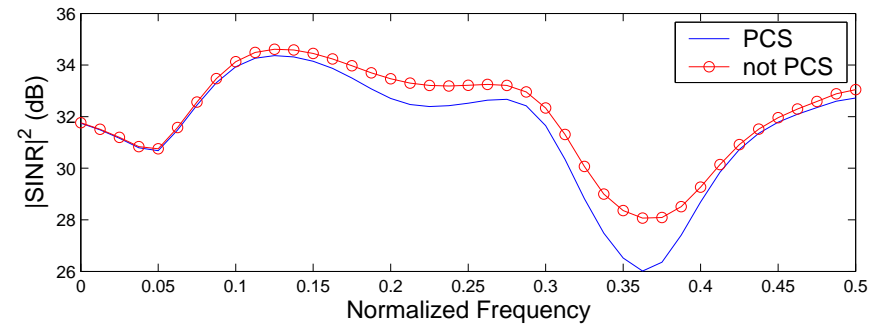

Figure 2: Comparative performance of the two schemes as the frequency of the narrow-band interferer is varied when $h^{(1)}(z)=z^{2}+2 z+3$ and $h^{(2)}(z)=7 z^{2}+2 z+1, \sigma^{2}=1$, and $g=\sqrt{2}$.

\section{Robustness to Interference: An Example}

We now consider a simple example including two types of interference: wide-band noise and a narrow-band interferer. In the case of the wide-band noise, $\mathbf{R}_{n}=\sigma^{2} \mathbf{I}$, where $\mathbf{I}$ is the identity matrix with all zero elements except for ones along the along the main diagonal [17]. When there is also a narrow-band interferer, one would expect that the channel shortener can stay a perfect channel shortener, but incorporate a notch into its response in order to block out the interferer. We consider a system in which the multi-channel model arises due to over-sampling the received signal by $P=2$. A narrow-band interferer of the form $g \cos (\Omega t)$ in the original continuous time channel gives the multi-channel interferers $\psi_{k}^{(i)}=g \cos (\omega(i-1+k P))$ where $\omega=\frac{\Omega T_{s}}{P}$ and $T_{s}$ is the transmitted symbol period. This yields the average matrix $\mathbf{R}_{\psi}$ whose elements are

$$
\mathbf{R}_{\psi}[i, j]=\frac{g^{2}}{2} \cos \left(\omega\left(2(i-j)-\left\lfloor i /\left(L_{w}+1\right)\right\rfloor+\left\lfloor j /\left(L_{w}+1\right)\right\rfloor\right)\right)
$$

As suspected, the simulations showed that the channel shortener's response attempted to notch out the narrow-band interferer for both schemes. Figures 2 and 3 compare the depth of this notch (the channel shorteners were normalized to have unit $L_{2}$ norm) for the schemes (7) and (8).

Another interesting aspect of the two designs is their robustness to inaccuracies in the measurement of the wide-band noise variance $\sigma^{2}$. We created Fig. 4 by removing the narrow-band interferer from the model, fixing the wide-band noise at $10 \mathrm{~dB}$ SNR and varying the mis-measured SNR from $5 \mathrm{~dB}$ to $15 \mathrm{~dB}$. It appears that, while the $\nu$-PCS is more robust against inaccurate SNR measurements than the imperfect channel shortener, both designs do not degrade in performance heavily with this impairment.

\section{CONCLUSION}

In this work we provided novel terminology and novel necessary conditions and recast sufficient conditions for perfect channel 


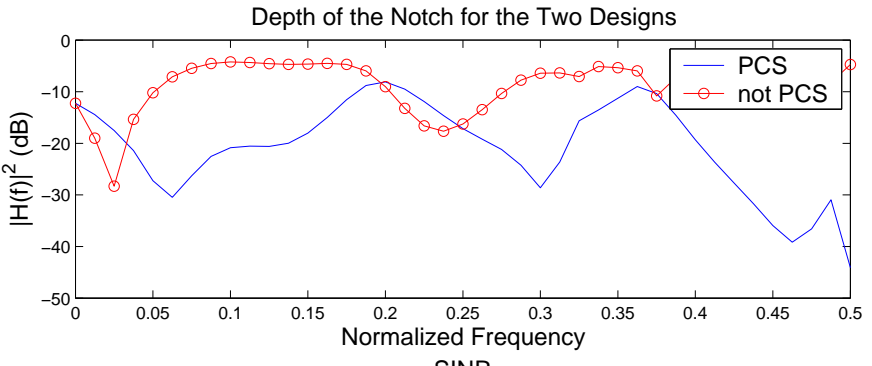

SINR

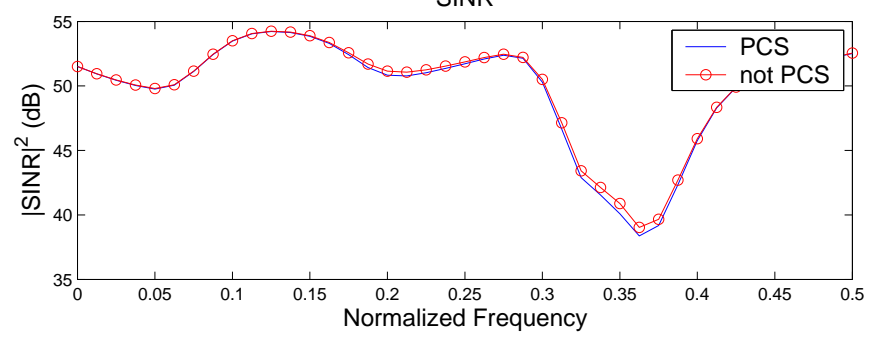

Figure 3: Comparative performance of the two schemes as the frequency of the narrow-band interferer is varied when $h^{(1)}(z)=z^{2}+2 z+3$ and $h^{(2)}(z)=7 z^{2}+2 z+1, \sigma^{2}=.1$, and $g=\sqrt{2}$.

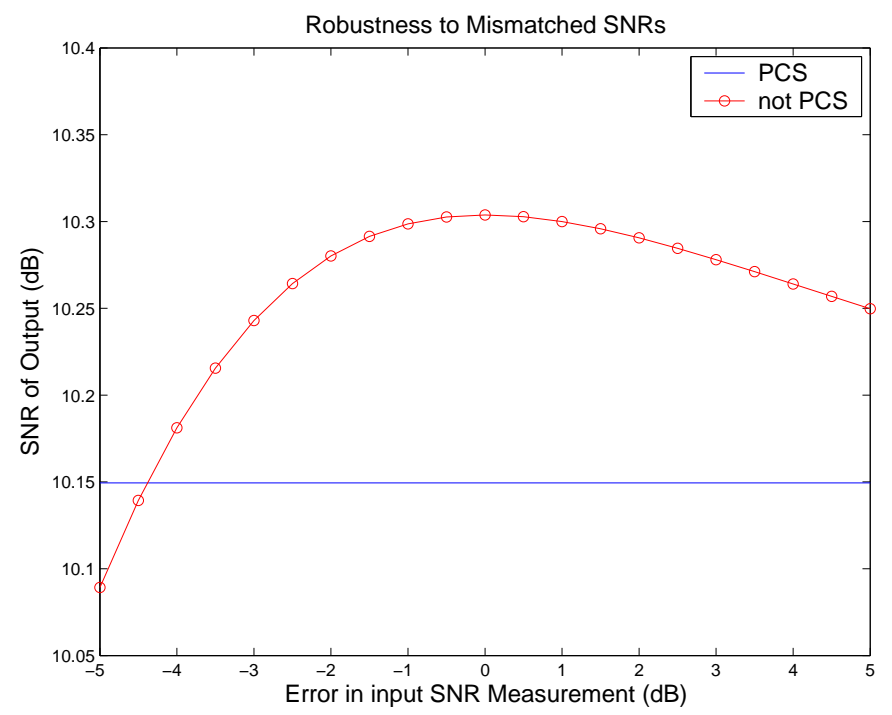

Figure 4: Performance of the two schemes when the noise is measured incorrectly.

shortening arising in a communications system exploiting multiple receive antennas or over-sampling. Any number of possible extensions to this work exist. Over time, quite a few schemes for channel shortening have been proposed, and one could check whether these schemes converge to PCSs under ideal noiseless conditions. Also, it would be interesting to investigate whether or not standard databases of channels, such as the CSA-loops for DMT systems satisfy the necessary and sufficient conditions for perfect channel shortening. Specifically, if these fractionally spaced channel models have sub-channels with nearly common roots, then over-sampling is not likely to provide the receiver a significant improvement in its ability to shorten the channel. Furthermore one could investigate the robustness of per- fect channel shorteners to interference resulting from cross-talk or investigate a truly MIMO channel model.

\section{ACKNOWLEDGMENTS}

The authors wish to thank Dr. D. Pal of Conexant, Dr. G. Iyengar of Columbia University, and Dr. J. Cioffi of Stanford University for providing us with [6] and [7]. We also would like to thank Dr. T. Miyajima of Ibaraki University and Dr. Z. Ding of U.C. Davis for providing us with [9].

\section{REFERENCES}

[1] P. J. W. Melsa, R. C. Younce, and C. E. Rohrs, "Impulse Response Shortening for Discrete Multitone Transceivers," IEEE Trans. on Comm., vol. 44, pp. 1662-1672, Dec. 1996.

[2] D. D. Falconer and F. R. Magee, "Adaptive Channel Memory Truncation for Maximum Likelihood Sequence Estimation," Bell Sys. Tech. Journal, pp. 1541-1562, Nov. 1973.

[3] S. Barbarossa, G. Scutari, and A. Swami, "MUI-free CDMA Systems Incorporating Space-Time Coding and Channel Shortening," in Proc. IEEE Int. Conf. on Acoustics, Speech, and Signal Processing, May 2002, vol. 3, pp. 2213-2216.

[4] D. Pal, G. N. Iyengar, and J. M. Cioffi, "A New Method of Channel Shortening With Applications to Discrete Multi Tone (DMT) Systems," in Proc. IEEE Int. Conf. on Comm., June 1998, vol. 2, pp. 763-768.

[5] D. Pal, "Poly-Path Time Domain Equalization," US patent no. 6,353,629, Mar. 2002.

[6] D. Pal, G. N. Iyengar, and J. M. Cioffi, "A New Method of Channel Shortening With Applications to Discrete Multi Tone (DMT) Systems. Part I: Theory," submitted to IEEE Trans. on Comm.

[7] D. Pal, G. N. Iyengar, and J. M. Cioffi, "A New Method of Channel Shortening With Applications to Discrete Multi Tone (DMT) Systems. Part II: Training," submitted to IEEE Trans. on Comm.

[8] T. Miyajima and Z. Ding, "Multicarrier Channel Shortening Based on Second-Order Output Statistics," in Proc. IEEE Workshop on Signal Proc. Advances in Wireless Comm., Rome, Italy, June 2003.

[9] T. Miyajima and Z. Ding, "Second-Order Statistical Approaches to Channel Shortening in Multicarrier Systems," to appear in IEEE Trans. on Signal Processing, 2004.

[10] M. Milosevic, L. F. C. Pessoa, and B. L. Evans, "Simultaneous Multichannel Time Domain Equalizer Design Based On The Maximum Composite Shortening SNR," in Proc. IEEE Asilomar Conf., Pacific Grove, CA, Nov. 2002, vol. 2, pp. 1895-1899.

[11] N. Al-Dhahir, "FIR Channel-Shortening Equalizers for MIMO ISI Channels," IEEE Trans. on Comm., vol. 49, no. 2, pp. 213218, Feb. 2001.

[12] W. Younis and N. Al-Dhahir, "Joint Prefiltering and MLSE Equalization of Space-Time Coded Transmissions Over Frequency-Selective Channels," IEEE Trans. on Vehicular Tech., vol. 51, pp. 144-154, Jan. 2002.

[13] A. Tkacenko and P. P. Vaidyanathan, "Eigenfilter Design of MIMO Equalizers for Channel Shortening," in Proc. IEEE Int. Conf. on Acoustics, Speech, and Signal Processing, Orlando, FL, May 2002, vol. 3, pp. 2361-2364.

[14] A. Stamoulis, S. N. Diggavi, and N. Al-Dhahir, "Intercarrier Interference in MIMO OFDM," IEEE Trans. on Signal Processing, vol. 50, no. 10, pp. 2451-2464, Oct. 2002.

[15] R. K. Martin, J. M. Walsh, and C. R. Johnson, Jr., "Low Complexity MIMO Blind, Adaptive Channel Shortening," Submitted to IEEE Trans. on Signal Processing, Oct. 3, 2003.

[16] C. R. Johnson, Jr., et. al., "The Core of FSE-CMA Behavior Theory," in Unsupervised Adaptive Filtering, vol. II: Blind Deconvolution, Simon Haykin, editor, pp. 13-112, Wiley, 2000.

[17] G. Strang, Linear Algebra and its Applications, Academic Press, Inc., New York, NY, 1980. 